\title{
Embryotoxic Effects of Brief Maternal Insulin-hypoglycemia during Organogenesis in the Rat
}

\author{
T. A. Buchanan, J. K. Schemmer, and N. Freinkel \\ Center for Endocrinology, Metabolism, and Nutrition, and Departments of Medicine and Molecular Biology and Biochemistry, \\ Northwestern University Medical School, Chicago, Illinois 60611
}

\section{Abstract}

To test whether maternal hypoglycemia can impair organogenesis, we induced brief glucopenia with insulin in conscious pregnant rats during either the headfold stage or the early neural tube closure stage of embryogenesis. At each time, 10 pairs of animals received identical insulin infusions for $1 \mathrm{~h}$. Half the animals were maintained at euglycemia during the infusions, while the others were allowed to become hypoglycemic. Euglycemia was maintained or restored in all animals immediately after the insulin was stopped. Spontaneous activity was diminished during the hypoglycemia but consciousness was preserved. Embryos were removed from mothers and examined $2 \mathrm{~d}$ later. This examination revealed that embryos from the hypoglycemic mothers were growth-retarded and displayed a small but significant incidence of gross developmental anomalies compared with embryos from the insulin-infused euglycemic mothers. Thus, brief, mild maternal hypoglycemia during early organogenesis can disrupt normal embryo development in the rat. The effect is due to the hypoglycemia per se rather than to the insulin employed for its induction.

\section{Introduction}

In previous studies, we have shown that postimplantation mammalian embryos depend on uninterrupted glycolysis for normal growth and development during early organogenesis; interference with glycolytic processes at this time resulted in generalized growth retardation as well as the production of developmental abnormalities of nervous, cardiovascular, skeletal, and other systems ("The Honeybee Syndrome" [1-3]). Our observations prompted the suggestion that seemingly unrelated environmental agents or events might be teratogenic by virtue of their ability to impair glycolysis in the conceptus at critical times in development $(1,2)$. This possibility may be particularly relevant to recent trends in the management of pregnancies complicated by diabetes mellitus. Physicians are now attempting very "tight"

Portions of this work were presented at the 58th Annual Meeting of the Central Society for Clinical Research, Chicago, IL, 1 November 1985 (published in abstract form, 1985. Clin. Res. 33:888A) and at the 46th annual meeting of the American Diabetes Association, Anaheim, CA, 24 June 1986 (Diabetes. 35:47A).

Address reprint requests to Dr. Buchanan, Center for Endocrinology, Metabolism, and Nutrition, Northwestern University Medical School, 303 E. Chicago Ave., Chicago, IL 60611.

Received for publication 11 February 1986 and in revised form 6 May 1986.

J. Clin. Invest.

(c) The American Society for Clinical Investigation, Inc.

0021-9738/86/09/0643/07 \$1.00

Volume 78, September 1986, 643-649 regulation of maternal diabetes during early pregnancy in an effort to reduce the incidence of birth defects in infants of diabetic mothers (4). This practice may increase the frequency of maternal hypoglycemic episodes during critical phases of organogenesis. We have previously proposed that such hypoglycemia during early pregnancy could impair development by limiting the availability of glucose for obligate glycolysis at critical points in embryogenesis (5). We now report that a relatively brief period of insulin-induced maternal hypoglycemia during early organogenesis in the rat does disrupt normal development and that this effect occurs independently of any direct embryotoxicity of insulin.

\section{Methods}

Animals. Virgin female rats of the Sprague-Dawley strain were obtained from Charles River Breeding Laboratories, Wilmington, MA. After at least $3 \mathrm{~d}$ of stabilization in an artificially lighted, controlled environment chamber (dark cycle, 1800-0500 h) with free access to a laboratory diet (Purina Rat Chow, Ralston Purina Co., St. Louis, MO) and tap water, females weighing $250 \pm 25 \mathrm{~g}$ were housed overnight with normal males of the same strain. Mating was confirmed by the presence of sperm in the vaginal smear the following morning. Midnight of the night of mating was designated day 0 of embryo development; the subsequent $24 \mathrm{~h}$ were considered the first day of gestation (6). Sperm-positive females were housed singly in cages with free access to water and laboratory diet except during infusions (see below). Animals and food were weighed daily between 0800 and $1000 \mathrm{~h}$ to assess the effects of experimental procedures on body weight and food consumption.

Infusion apparatus. Techniques employed for the placement of indwelling jugular blood sampling catheters and tail vein infusion catheters have been described in detail elsewhere (3). Briefly, between 1600 and $1800 \mathrm{~h}$ on the seventh day of gestation (day 6.7 of embryo development), each animal underwent placement of a sterile polyethelene catheter into the right internal jugular vein during light ether anesthesia. Catheters were exteriorized over the occiput, filled with heparin solution (100 U/ $\mathrm{ml}$ in $0.9 \%$ saline), and flushed twice daily to maintain patency. These catheters permitted the sampling of mixed venous blood from unrestrained animals.

Between 0700 and $0900 \mathrm{~h}$ on the 10th day of gestation (day 9.3 of embryo development), additional sterile polyethelene catheters were placed in both lateral tail veins while animals remained conscious but were briefly restrained in towels. The distal portions of the tail and the infusion catheters were then drawn through a small hole in the side of each animal's cage, fixed there with adhesive tape, and animals were released from their restraints. This arrangement provided separate portals for the infusion of glucose and insulin while allowing animals the freedom to move about during experiments.

Insulin infusions. Primed ( $45 \mathrm{mU} / \mathrm{kg}$ body weight) continuous (10 $\mathrm{mU} / \mathrm{kg}$ body weight/min) infusions of crystalline human insulin ( $\mathrm{Hu}-$ mulin; Eli Lilly \& Co., Indianapolis, IN) were administered simultaneously to pairs of 12-h fasted gravida between 1200 and $1300 \mathrm{~h}(n$ $=10$ pairs) or between 1800 and $1900 \mathrm{~h}$ ( $n=10$ pairs) on the 10th day of gestation (day 9.5 and 9.75 of embryo development, respectively). Infusions were delivered for $60 \mathrm{~min}$ via one tail vein catheter by an infusion pump (model 975; Harvard Apparatus Co., S. Natick, MA) and 
were designed to produce "square-wave" hyperinsulinemia. Plasma samples were obtained via jugular catheters before and at 10 -min intervals during and for $80 \mathrm{~min}$ after the insulin infusions. These samples were analyzed immediately for glucose (Beckman Glucose Analyzer II; Beckman Instruments, Inc., Fullerton, CA) and the 0-, 20-, 40-, 60-, 80-, $100-, 120$, and $140-\mathrm{min}$ specimens were stored at $-15^{\circ} \mathrm{C}$ for subsequent analyșis.

One animal in each pair (designated "euglycemic") received an infusion of dextrose (10\% wt/vol in water) via the remaining tail vein catheter beginning $4 \mathrm{~min}$ after the start of the insulin infusion. Dextrose infusions were initiated at a rate of $30 \mathrm{mg} / \mathrm{kg}$ body weight/min and were adjusted at 10-min intervals, based on plasma glucose measurements, to maintain plasma glucose at preinfusion levels. The other animal in each pair (designated "hypoglycemic") received no dextrose during the 1-h insulin infusion, but was returned to preinfusion glycemic levels with a variable-rate infusion of dextrose starting at the end of the insulin infusion. Dextrose infusions were continued in all animals until plasma glucose remained stable in the absence of exogenous dextrose (within $40 \mathrm{~min}$ after the termination of insulin infusions in all cases). Animals remained in tail restraints with free access to food and water after the infusions.

Examination of embryos. At $1400 \mathrm{~h}$ on the 12th day of gestation (day 11.6 of embryo development) gravida were sacrificed by cervical dislocation. Uteri were excised rapidly, rinsed, and immersed at room temperature in $20 \mathrm{ml}$ of $0.9 \%$ saline contained within a petri dish. Individual "embryo units" consisting of the embryo and associated membranes were freed of surrounding decidua and introduced into a second petri dish containing $20 \mathrm{ml}$ of saline. Embryos and investing membranes were teased apart with fine jewelers' forceps during visualization with a stereomicroscope (Model MSA; Wild Heerbrugg Instruments, Inc., Farmingdale, NY) and the total number of somites and the crown-rump length (the maximum length of the embryo in its natural position [7]) were determined for each embryo. Embryos were then individually inspected to determine whether the morphology of the brain spheres, neural tube, heart, optic and otic vesicles, limb buds, branchial arches, and axial curvature conformed to that expected at day 11.6 of development (7). Embryos not conforming to this normal morphology in any of the above structures were considered dysmorphic. After visual inspection, individual embryos were introduced into $0.5 \mathrm{~N} \mathrm{NaOH}$ for the subsequent determination of total protein $(8)$ and DNA $(9,10)$ content. These determinations were performed by an individual who was unaware of the conditions (i.e., euglycemia or hypoglycemia) to which the embryos had been exposed.

Materials and analytical methods. Insulin for infusions was diluted in a sterile solution containing $0.01 \mathrm{ml}$ of autologous plasma per milliliter of $0.9 \%$ saline. Plasma immunoreactive insulin (IRI) ${ }^{1}$ concentrations were determined by double antibody radioimmunoassay using human insulin standard (Novo Laboratories, Bagsvaard, Denmark) and guinea pig anti-beef insulin antibody. Plasma samples from matched pairs of animals were assayed simultaneously in all instances. Plasma $\beta$-hydroxybutyrate concentrations were determined enzymatically (11) after specimens were deproteinized with perchloric acid.

Statistical analysis. All data are presented as group mean values plus or minus one standard error. Intergroup differences in the prevalence of morphologic lesions and resorbed embryos were assessed by Fisher's exact test or chi-square analysis. All other comparisons were performed using unpaired $t$ tests.

\section{Results}

Plasma glucose and IRI. Plasma glucose and IRI levels that were observed during the 60 -min insulin infusions and for 60 min thereafter on days 9.5 and 9.75 of gestation are summarized in the left and right panels of Fig. 1, respectively.

In the absence of exogenous dextrose, glucose levels in an-

1. Abbreviations used in this paper: IRI, immunoreactive insulin. imals studied on day 9.5 of development (Fig. 1, left top) fell rapidly from a preinfusion mean of $141 \pm 2 \mathrm{mg} / \mathrm{dl}$ to $53 \pm 5 \mathrm{mg} /$ dl within $20 \mathrm{~min}$ after the start of the insulin infusions. Glucose then declined more gradually to $42 \pm 2 \mathrm{mg} / \mathrm{dl}$ by the end of the 60-min insulin infusions. In the pair-matched euglycemic animals, variable-rate dextrose infusions kept plasma glucose approximately at preinfusion levels throughout the same $60-\mathrm{min}$ period. Upon discontinuation of the insulin infusions, exogenous dextrose was used to restore hypoglycemic animals to approximately preinfusion glycemic levels $(145 \pm 5 \mathrm{mg} / \mathrm{dl})$ within $20 \mathrm{~min}$ and to maintain them there until exogenous dextrose was no longer required. Virtually identical plasma glucose levels were maintained in euglycemic animals after the insulin infusions were stopped.

Similar patterns for plasma glucose were observed in the pair-matched animals studied on day 9.75 of embryo development (Fig. 1, right top). In the hypoglycemic group, insulin produced a prompt and sustained fall in maternal plasma glucose to $54 \pm 4 \mathrm{mg} / \mathrm{dl}$ and $45 \pm 2 \mathrm{mg} / \mathrm{dl}$ after 20 and $60 \mathrm{~min}$ of insulin administration, respectively. This hypoglycemia was reversed by exogenous dextrose (to $142 \pm 5 \mathrm{mg} / \mathrm{dl}$ ) within $20 \mathrm{~min}$ after the insulin was stopped. In contrast, plasma glucose in the euglycemic group was maintained approximately at preinfusion levels both during and after the insulin infusions.

Concurrent plasma IRI values are depicted in the bottom panels of Fig. 1. The primed, 60 -min insulin infusions effected essentially square-wave elevations of IRI in maternal plasma. On day 9.5 of gestation, plasma IRI levels during the insulin infusions averaged $315 \pm 11 \mu \mathrm{U} / \mathrm{ml}$ in the hypoglycemic animals and $290 \pm 15 \mu \mathrm{U} / \mathrm{ml}$ in the euglycemic group (Fig. 1; left bottom). Maternal plasma IRI during insulin infusions on day 9.75 of gestation averaged $318 \pm 27 \mu \mathrm{U} / \mathrm{ml}$ and $318 \pm 17 \mu \mathrm{U} / \mathrm{ml}$ in the hypoglycemic and euglycemic gravida, respectively (Fig. 1, right top). In all groups, maternal IRI levels returned to preinfusion values within $20 \mathrm{~min}$ after the insulin infusions were stopped.

$\beta$-hydroxybutyrate concentrations were measured in plasma from six of the hypoglycemic mothers, three from the day 9.5 group and three from the day 9.75 group. Both during (20-min and 60-min samples) and after (80-min, 100-min, and 140-min samples) the insulin infusions, the levels were uniformly $<1.3$ $\mathrm{mmol} /$ liter, which is well below the $\beta$-hydroxybutyrate concentrations that we (12) and others (13) have found to be teratogenic to rat embryos in vitro.

Behavioral changes were observed only in the two hypoglycemic groups. Within 20 min after the start of the insulin infusions, hypoglycemic gravida manifested decreased alertness and diminished spontaneous activity. The changes were mild and similar on days 9.5 and 9.75 of gestation. No animals displayed seizure activity and all remained awake and responsive to tactile stimuli. These behavioral changes persisted for 10-20 min after the discontinuation of insulin infusions. At that time, plasma glucose had been restored to preinfusion levels with exogenous dextrose and all animals resumed normal activity patterns.

Maternal nutrition did not appear to be affected by the brief interval of hypoglycemia. Mean values for daily food intake and body weight change between days 9.5 and 11.6, i.e., the interval during and after the insulin infusions, were not significantly different between groups of pair-infused euglycemic and hypoglycemic mothers (Table I).

Analysis of embryos. Hysterotomy was performed on day 11.6 of gestation to evaluate embryos from mothers that had 

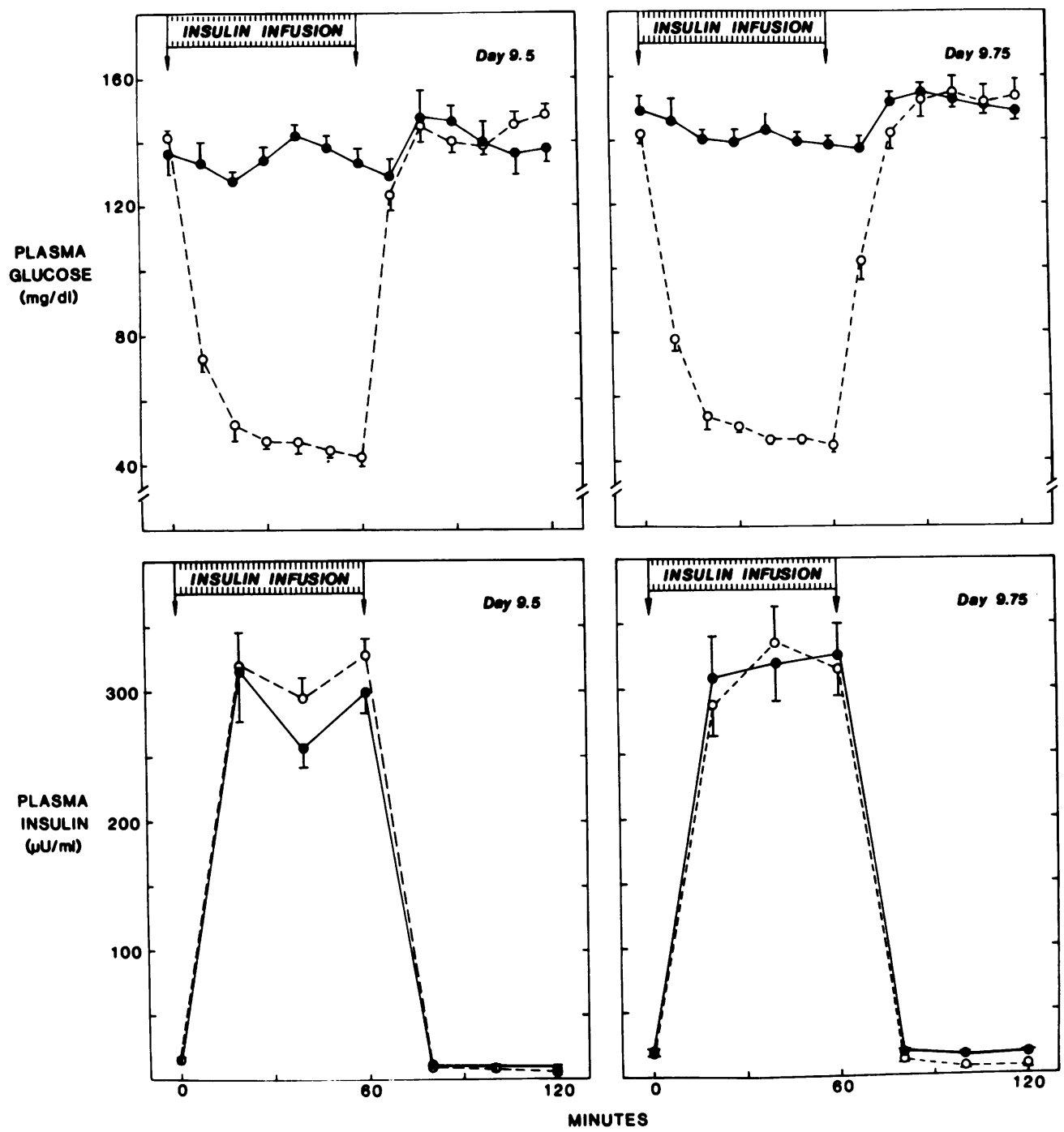

Figure 1. Plasma glucose (top panels) and immunoreactive insulin (bottom panels) concentrations during and after 60-min infusions of insulin (hatched bars) into pregnant rats. Infusions were initiated at day 9.5 (0 $\mathrm{min}$; left panels) or day 9.75 (0 min; right panels) of embryo development. "Euglycemic" animals (- -$)$ received dextrose infusions during and after insulin; "hypoglycemic" animals (-० - ) received dextrose only after insulin, as described in the text.

been infused with insulin for $60 \mathrm{~min}$ on day 9.5 or day 9.75 . Morphological and compositional characteristics are summarized in Table II.

Table I. Maternal Food Intake and Body Weight Change Between Days 9.5 and 11.6 of Embryo Development*

\begin{tabular}{ll}
\hline Food intake & $\Delta$ Body weight \\
\hline$g / d$ & $g / d$
\end{tabular}

Day 9.5

$\begin{array}{lll}\text { Euglycemic (10) } & 13.9 \pm 0.8 & 1.1 \pm 0.9 \\ \text { Hypoglycemic (10) } & 15.9 \pm 1.5 & 1.4 \pm 0.7 \\ \text { Day 9.75 } & & \\ \text { Euglycemic (10) } & 14.6 \pm 1.3 & -2.2 \pm 0.9 \\ \text { Hypoglycemic (10) } & 14.3 \pm 1.2 & -1.4 \pm 1.1\end{array}$

* Mean \pm SE values obtained by weighing animals and food daily. " $\Delta$ Body weight" refers to change in body weight. "Day 9.5" and "Day 9.75" refer to the stage of embryo development at which 60-min insulin infusions were begun into paired "euglycemic" and "hypoglycemic" pregnant rats, as described in text. Numbers in parentheses denote the number of pregnant animals in each group. None of the differences between groups was statistically significant (all $P>0.05$ ).
10 euglycemic and 10 hypoglycemic animals studied on day 9.5 of development provided 148 and 138 embryos, respectively. Mean litter sizes (14.8 \pm 0.6 vs. $13.8 \pm 1.1)$ and the total number of resorbed conceptuses ( 7 vs. 6 ) were not significantly different between these two groups. However, morphometric examinations disclosed significantly lower values for crown-rump length and somite number in the embryos from the hypoglycemic group; compositional analyses revealed significantly lower values for total protein and total DNA content as well (Table II). That these differences reflected developmental retardations in the hypoglycemic group rather than accelerated growth in the euglycemic group was corroborated by examining embryos on day 11.6 from mothers that had received no treatment of any kind ("uninfused"; Table II). Values for crown-rump length, somite number, total protein, and total DNA in 107 embryos from eight uninfused dams were indistinguishable from those observed in the embryos from the euglycemic mothers, but were significantly greater than values in the embryos that had been exposed to $60 \mathrm{~min}$ of maternal hypoglycemia on day 9.5 (Table II). In addition to this generalized retardation in growth, maternal hypoglycemia on day 9.5 was associated with grossly anomalous development in three embryos. These embryos were from three separate dams and uniformly exhibited failure of neural tube closure, gross deformities of brain sphere formation, and ab- 
Table II. Somite Number, Crown-Rump Length, Total Protein, and Total DNA of

11.6-d Rat Embryos from Uninfused, Euglycemic, and Hypoglycemic Mothers

\begin{tabular}{|c|c|c|c|c|c|}
\hline Maternal group & Embryos & Somites & Crown-Rump & Protein & DNA \\
\hline & $n$ & \# & $\mathrm{mm}$ & $\mu g$ & $\mu g$ \\
\hline Uninfused (8) & 107 & $30.3 \pm 0.1$ & $4.13 \pm 0.01$ & $400 \pm 5$ & $52.0 \pm 0.8$ \\
\hline \multicolumn{6}{|l|}{ Day 9.5} \\
\hline Euglycemic (10) & 148 & $30.1 \pm 0.1$ & $4.11 \pm 0.02$ & $395 \pm 6$ & $50.7 \pm 0.8$ \\
\hline Hypoglycemic (10) & 138 & $29.1 \pm 0.2^{*} \ddagger$ & $3.92 \pm 0.03^{*} \ddagger$ & $346 \pm 6^{*} \ddagger$ & $44.7 \pm 0.9^{*} \ddagger$ \\
\hline \multicolumn{6}{|l|}{ Day 9.75} \\
\hline Euglycemic (10) & 133 & $30.5 \pm 0.1$ & $4.19 \pm 0.03$ & $416 \pm 7$ & $53.4 \pm 1.1$ \\
\hline Hypoglycemic (10) & 137 & $28.9 \pm 0.2^{*} \ddagger$ & $3.87 \pm 0.04^{*} \ddagger$ & $332 \pm 7^{*} \ddagger$ & $40.9 \pm 1.1^{*} \ddagger$ \\
\hline
\end{tabular}

Mean \pm SE values at analysis on day 11.6 of embryo development. Uninfused animals received no prior treatment of any kind. "Day 9.5", "Day 9.75", and numbers in parentheses are as defined in Table I. ${ }^{*} P<0.001$ vs. euglycemic group infused at the same developmental stage.

$\ddagger P<0.001$ vs. uninfused group.

normal axial rotation and cardiac differentiation. Two of the three displayed grossly deranged optic vesicle and somite development as well. All embryos from the euglycemic group of animals infused on day 9.5 were normally developed at day 11.6.

Findings were similar in embryos from mothers infused at day 9.75 of development. Neither mean litter sizes $(13.3 \pm 1.2$ vs. $13.7 \pm 0.8$ ) nor the total number of resorbed conceptuses ( 4 vs. 3) differed between euglycemic and hypoglycemic groups. Values for crown-rump length, somite number, total protein, and total DNA in 137 embryos from 10 hypoglycemic mothers were significantly reduced when compared to these parameters in 133 embryos from the 10 euglycemic control animals or in the 107 embryos from the uninfused mothers (Table II). Dysmorphogenic changes were present in nine embryos from five of the hypoglycemic mothers but in only one embryo from a euglycemic mother $(P<0.02)$. In four of the nine, the abnormality was limited to a persistently open posterior neuropore. This finding could have been consistent with the degree of developmental delay seen in each case, since the posterior neuropore is the last portion of the neural tube to close. However, the other five embryos displayed morphological features that are not encountered during the normal developmental sequence in the rat. Therefore, these features could not be explained by developmental retardation alone. They included microencephaly and malformed brain spheres (four embryos), abnormal somite development (three embryos), abnormal axial rotation (two embryos), persistent opening of the anterior neuropore in the presence of a closed posterior neuropore (one embryo), and "squirrellike" (14) fusion of the anterior and posterior neural folds (one embryo). The single abnormal embryo from the euglycemic group was found to have a circumferential constriction at the level of the anterior limb-bud with hypoblasia of structures posterior to this. Brain, cardiac, and neural tube development and axial rotation were normal in this embryo.

Examination of frequency distribution plots for embryo total protein and DNA suggested that some degree of growth retardation was a generalized phenomenon in the embryos exposed to maternal hypoglycemia. These plots were distributed in a Gaussian fashion in all four experimental groups, but were shifted toward lower protein and DNA values in the hypoglycemic groups compared with their respective euglycemic controls. Thus, it appeared that few of the embryos from hypoglycemic mothers had reached their expected growth by day 11.6 of development.

To assess whether the vulnerabilities to a brief hypoglycemic insult were different on day 9.5 than on day 9.75 of development, we compared litter mean values for total embryo protein between matched pairs of euglycemic and hypoglycemic mothers. The average reductions in total protein between matched litters tended to be greater after hypoglycemia on day 9.75 than on day $9.5(19.3 \pm 4.2 \%$ vs. $11.2 \pm 2.8 \%$ ), although this difference did not achieve statistical significance $(P>0.05)$. Similarly, the seemingly greater incidence of embryos with dysmorphogenesis in the day $9.75(9 / 137)$ than in the day $9.5(3 / 138)$ hypoglycemic group was not statistically significant $(P>0.05)$.

\section{Discussion}

In these studies, transitory hypoglycemia was induced in pregnant rats at two points in early organogenesis. Day 9.5 of gestation was selected as a time at which the neural plate is established in the postimplantation rat embryo but vascularization of the yolk sac has not yet been initiated; day 9.75 was chosen as a somewhat later stage when somites are forming, neural folds are beginning to appose, and vascular islands are appearing in the yolk sac. Previous studies from this laboratory have shown that normal development is largely dependent upon uninterrupted glycolysis at both of these phases of embryogenesis (13). Accordingly, we felt that they might constitute suitable time points for testing whether maternal hypoglycemia can impair the normal developmental sequence in the rat embryo. To accomplish this, we used insulin infusions by square-wave delivery to effect circumscribed periods of hypoglycemia in mothers. We simultaneously maintained paired insulin-infused control animals at normoglycemia with exogenous dextrose to test for any potential teratogenic effects of insulin per se (15-19). We limited the duration of the hypoglycemia to $1 \mathrm{~h}$ and opted for a mild glucopenia that would not disrupt maternal consciousness in order to mimic the type of hypoglycemia that may occur during clinical attempts at strict diabetes control. Finally, we examined embryos $\sim 2 \mathrm{~d}$ after the hypoglycemic exposure to maximize 
the likelihood of detecting any modifications of normal embryogenesis.

Within this framework, we found unequivocal evidence that mild insulin-mediated maternal hypoglycemia can impair the growth and development of the early postimplantation rodent embryo. We observed a small incidence of developmental anomalies in association with a generalized retardation of growth on day 11.6 of development in embryos from mothers rendered hypoglycemic for $1 \mathrm{~h}$ on day 9.5 or day 9.75 . Although we do not know the extent to which these phenomena will persist until birth, the occurrence at day 11.6 of sporadic dysmorphogenesis in a setting of overall impaired growth is consistent with our previous experiences with fuel-mediated teratogenesis $(1-3,20)$ and with the suggestions of others $(21,22)$. In our present experiments, neither the magnitude of the growth retardation nor the frequency of malformations was statistically different between the embryos exposed to maternal hypoglycemia during the head fold stage (day 9.5) and those exposed during the early apposition of neural folds (day 9.75). Thus, we could not document any developmental stage specificity for the abnormalities induced by maternal hypoglycemia during the intervals that we studied. Further investigation will be required to determine whether a similar vulnerability to glucopenic insults is present during other phases of embryogenesis.

The proximate cause of the embryotoxicity that we observed in association with maternal hypoglycemia cannot be ascertained from the present experiments. Clearly, insulin itself was not the cause since plasma insulin levels did not differ between euglycemic and hypoglycemic mothers studied at the same developmental phase. Likewise, daily food intake and weight gain were similar between these groups, so that differences in maternal nutrition cannot be invoked to explain the developmental abnormalities that we observed. Based on our previous studies (13 ) and the in vitro experiences of others with rodent embryos cultured in glucose-depleted media $(23,24)$, we would suggest that these abnormalities were due to the briefly diminished availability of glucose per se. However, we cannot exclude the possibility that maternal counterregulatory responses to hypoglycemia may have been involved as well. Nonetheless, since each of the latter is part of the normal physiological response to intracellular glucopenia in mammals (25-28), the maternal hypoglycemia must be deemed the initiating event and hence a real risk factor during early organogenesis.

Previous attempts to evaluate the effects on the conceptus of maternal insulin-induced hypoglycemia during early mammalian pregnancy have entailed prolonged and severe glucopenia. Thus, the developmental defects that have been reported after the administration of protamine zinc insulin to rats $(29$, 30 ) or mice (31) or pharmacological doses of insulin to rabbits (32) during early pregnancy occurred in association with seizures $(31,32)$, deaths $(29,31,32)$, and prolonged (i.e., up to $36 \mathrm{~h}[30])$ hypoglycemia in mothers. The relevance of those observations to the generally mild and evanescent hypoglycemia that occurs during clinical attempts at strict diabetes control has therefore been unclear. Furthermore, none of these earlier studies evaluated the potential for direct effects of insulin on the conceptus, which are of some concern in view of the embryotoxic actions that have been ascribed to insulin in the chick (15-19). Our experimental design enabled us to overcome these limitations and thereby to demonstrate for the first time that a relatively mild and brief episode of maternal insulin-induced hypoglycemia during early mammalian organogenesis may be embryotoxic, and that this phenomenon is independent of any direct toxic effects of insulin.

Since studies analogous to the present one cannot be performed in human pregnancy and may be logistically prohibitive in subhuman primates, it becomes important to determine if our findings may be extrapolated to clinical practice. Clearly, a definitive answer cannot be provided. Indeed, previous clinical analyses have failed to disclose an increased incidence of birth defects in the infants of mothers sustaining episodes of insulininduced hypoglycemia during pregnancy (33-36). Those reports need not be inconsistent with the findings of the present study. We have focused on the interval during rat embryogenesis between the beginning of neurulation, when nutrient delivery to the conceptus must occur by simple diffusion, and the establishment of the yolk sac and allantoic placental circulations, when more direct access to the maternal circulation presumably becomes possible. Previous studies have shown that development in the rat is primarily dependent on glycolysis during this period $(1-3,37-40)$. Although an analogous glycolytic dependence has not been clearly established in human embryos, comparable developmental events occur in humans between day 18 (beginning of neurulation) and day 22 (placental vascularization and initial circulation) (41-43). Thus, if human embryos are likewise susceptible to glycolytic interruption at this phase of embryogenesis, the period of vulnerability may be maximal during the latter part of the third week of development. Previous clinical studies which have not focused on hypoglycemia occurring during this interval may have overlooked an embryotoxic effect, although appropriate clinical data may be forthcoming from the ongoing Diabetes in Early Pregnancy Study (44).

For the moment and in light of the above, a reappraisal of recent clinical approaches to the management of diabetes in early pregnancy may be appropriate. Although poorly controlled maternal diabetes has been linked to birth defects in offspring both clinically (45-47) and experimentally (48-50), the extent to which metabolic control must be improved to mitigate this teratogenesis has not been determined. Miller et al. (46) and Ylinen et al. (47) failed to detect an increased incidence of birth defects in infants whose mothers had mildly elevated hemoglobin $A_{1 c}$ levels near the end of the first trimester of pregnancy (up to $8.5 \%$ of the total hemoglobin in the former study and up to $7.3 \%$ in the latter); the risk of birth defects was increased in association with higher glycohemoglobin levels in both studies. These findings, together with emerging clinical experiences (4), support the concept that improved diabetic control during early pregnancy may reduce the risk of birth defects in infants but they do not indicate that a complete normalization of maternal metabolism will be required to achieve this goal. Nonetheless, if hypoglycemia posed no risk to embryos, then attempts to fully normalize blood sugar levels during early pregnancy might seem justified until the precise degree of metabolic control that will be necessary to prevent birth defects has been defined. However, the present study clearly shows that a relatively brief period of maternal hypoglycemia is indeed embryotoxic in at least one mammalian species. Thus, although our findings cannot be directly extrapolated to humans, they do suggest that a cautious approach to improved blood sugar regulation during early pregnancy may well constitute the best therapeutic course of action for all who, like ourselves, favor "meticulous control" (28) at all other time points in pregnancy complicated by diabetes mellitus. 


\section{Acknowledgments}

The authors are indebted to Frances Novak, Francesca Brutto, and Leticia Guzman for their excellent help in the preparation of this manuscript.

This study was supported in part by research grants AM-10699, MRP HD-11021, and training grant AM-07169 from the National Institutes of Health. Dr. Buchanan was supported in part by a National Research Service Award from the National Institutes of Health (AM-07008), by a Clinical Research Fellowship of the Chicago Community Trust, and by a Young Investigator Research Award from the Northern Illinois Affiliate of the American Diabetes Association.

\section{References}

1. Freinkel, N., N. J. Lewis, S. Akazawa, L. Gorman, and M. Potaczek. 1983. The honeybee syndrome: teratogenic effects of mannose during organogenesis in rat embryo culture. Trans. Assoc. Am. Physicians. 96: 44-55.

2. Freinkel, N., N. J. Lewis, S. Akazawa, S. I. Roth, and L. Gorman. 1984. The honeybee syndrome-implications of the teratogenesis of mannose in rat-embryo culture. $N$. Engl. J. Med. 310:223-230.

3. Buchanan, T., N. Freinkel, N. J. Lewis, B. E. Metzger, and S. Akazawa. 1985. Fuel-mediated teratogenesis: use of D-mannose to modify organogenesis in the rat embryo in vivo. J. Clin. Invest. 75:1927-1934.

4. Fuhrmann, K., H. Reicher, K. Semmler, F. Fischer, M. Fischer, and E. Glöckner. 1983. Prevention of congenital malformations in infants of insulin-dependent diabetic mothers. Diabetes Care. 6:219-223.

5. Freinkel, N., S. L. Dooley, and B. E. Metzger. 1985. Care of the pregnant woman with insulin-dependent diabetes mellitus. $N$. Engl. J. Med. 313:96-101.

6. Kalter, H. 1968. How should times during pregnancy be called in teratology? Teratology. 1:231-234.

7. Brown, N. A., and S. Fabro. 1981. Quantitation of rat embryonic development in vitro: a morphological scoring system. Teratology. 24: 65-78.

8. Lowry, O. H., N. J. Rosebrough, A. L. Farr, and R. J. Randall. 1951. Protein measurement with the Folin phenol reagent. J. Biol. Chem. 193:265-275.

9. Kissane, J. M., and E. Robbins. 1958. The fluorometric measurement of deoxyribonucleic acid in animal tissues with special reference to the central nervous system. J. Biol. Chem. 233:184-188.

10. Hinegardner, R. T. 1971. An improved fluorometric assay for DNA. Anal. Biochem. 39:197-201.

11. Williamson, D. H., and J. Mellanby. 1974. D(-)-3-hydroxybutyrate. In Methods of Enzymatic Analysis. H. D. Bergmeyer, editor. Academic Press, Inc., New York. 1836-1839.

12. Lewis, N. J., S. Akazawa, and N. Freinkel. 1983. Teratogenesis from $\beta$-hydroxybutyrate during organogenesis in rat embryo organ culture and enhancement by subteratogenic glucose. Diabetes. 32:41A. (Abstr.)

13. Horton, W. E., Jr., and T. W. Sadler. 1983. Effects of maternal diabetes on early embryogenesis: alterations in morphogenesis produced by the ketone body $\beta$-hydroxybutyrate. Diabetes. 32:610-616.

14. Cockroft, D. L., and P. T. Coppola. 1977. Teratogenic effects of excess glucose on headfold rat embryos in culture. Teratology. 16:141146.

15. Landauer, W. 1945. Rumpleness of chicken embryos produced by the injections of insulin and other chemicals. J. Exp. Zool. 98:6577.

16. Chen, J. H. 1954. The effect of insulin on embryonic limb bone cultivated in vitro. J. Physiol. 125:148-162.

17. Zwilling, E. 1959. Micromelia as a direct effect of insulin. Evidence from in vitro and in vivo experiments. J. Morph. 104:159-179.

18. Landauer, W. 1972. Is insulin a teratogen? Teratology. 5:129136.

19. de Pablo, F., E. Hernandez, F. Collia, and J. A. Gomez. 1985.
Untoward effects of pharmacological doses of insulin in early chick embryos: through which receptor are they mediated? Diabetologia. 28:308313.

20. Eriksson, U. J., N. J. Lewis, and N. Freinkel. 1984. Growth retardation during early organogenesis in embryos of experimentally diabetic rats. Diabetes. 33:281-284.

21. Pedersen, J. F., and L. Mølsted-Pedersen. 1981. Early fetal growth delay detected by ultrasound marks increased risk of congenital malformation in diabetic pregnancy. Br. Med. J. 283:269-271.

22. Spiers, P. S. 1982. Does growth retardation predispose the fetus to congenital malformation? Lancet. i:312-314.

23. Ellington, S. K. L. 1980. In vivo and in vitro studies on the effects of maternal fasting during embryonic organogenesis in the rat. J. Reprod. Fert. 60:383-388.

24. Sadler, T. W., and W. E. Horton, Jr. 1983. Effects of maternal diabetes on early embryogenesis: the role of insulin and insulin therapy. Diabetes. 32:1070-1074.

25. Freinkel, N., B. E. Metzger, E. Harris, S. Robinson, and M. Mager. 1972. The hypothermia of hypoglycemia. Studies with 2-deoxy-D-glucose in normal human subjects and mice. N. Engl. J. Med. 287:841-845.

26. Garber, A. J., P. E. Cryer, J. V. Santiago, M. W. Haymond, A. S. Pagliara, and D. M. Kipnis. 1976. The role of adrenergic mechanisms in the substrate and hormonal response to insulin-induced hypoglycemia in man. J. Clin. Invest. 58:7-15.

27. Phillipe, M., and J. L. Kitzmiller. 1981. The fetal and maternal catecholamine response to insulin-induced hypoglycemia in the rat. Am. J. Obstet. Gynecol. 139:407-415.

28. Unger, R. H. 1982. Special Comment: Meticulous control of diabetes: benefits, risks, and precautions. Diabetes. 31:479-483.

29. Lichtenstein, H., G. M. Guest, and J. Warkany. 1951. Abnormalities of offspring of white rats given protamine zinc insulin during pregnancy. Proc. Soc. Exp. Biol. Med. 78:398-402.

30. Hannah, R. S., and K. L. Moore. 1971. Effects of fasting and insulin on skeletal development in rats. Teratology. 4:135-140.

31. Smithberg, M., and M. N. Runner. 1963. Teratogenic effects of hypoglycemic treatments in inbred strains of mice. Am. J. Anat. 113: 479-489.

32. Chomette, G. 1955. Entwicklungsstörungen nach insulinschock beim trächtigen kaninchen. Beitr. Path. Anat. 115:439-451.

33. Melsted-Pedersen, L., I. Tygstrup, and J. Pedersen. 1964. Congenital malformations in newborn infants of diabetic women. Lancet. i: 1124-1126.

34. Rowland, T. W., J. P. Hubbell, and A. S. Nodas. 1973. Congenital heart disease in infants of diabetic mothers. J. Pediatr. 83:815-820.

35. Chung, C. S., and N. C. Myrianthopoulos. 1976. Factors affecting risks of congenital malfunctions. II. Effect of maternal diabetes. In Birth Defects: Original Article Series. Vol. XI, No. 10. D. Bergsma, editor. Symposia Specialists, Miami, FL. 23-27.

36. Pedersen, J. 1977. The Pregnant Diabetic and her Newborn. Problems and Management, 2nd Edition. Munksgaard, Copenhagen. 191-197.

37. Shepard, T. H., T. Tanimura, and M. A. Robkin. 1970. Energy metabolism in early mammalian embryos. Dev. Biol. Suppl. 29:42-58.

38. Tanimura, T., and T. H. Shepard. 1970. Glucose metabolism by rat embryos in vitro. Proc. Soc. Exp. Biol. Med. 135:51-54.

39. Neubert, D., H. Peters, S. Teske, E. Köhler, and H.-J. Barrach. 1971. Studies on the problem of "aerobic glycolysis" occurring in mammalian embryos. Naunym-Schmiedebergs Arch. Pharmak. 268:235-241.

40. Spielman, H. R., R. Meyer-Wendeker, and F. Spielman. 1973. Influence of 2-deoxy-D-glucose and sodium fluoroacetate on respiratory metabolism of rat embryos during organogenesis. Teratology. 7:127134.

41. Arey, L. B. 1954. Developmental Anatomy. W. B. Saunders Co., Philadelphia, PA. 119-150 and 340-392.

42. Wilkin, P. G. 1965. Organogenesis of the human placenta. In 
Organogenesis. R. L. DeHaan and H. Ursprung, editors. Holt, Rinehart \& Winston, New York. 743-768.

43. Pansky, B. 1982. Review of Medical Embryology. Macmillan Publishing Co., New York. 44-56.

44. Mills, J. L., A. R. Fishl, R. H. Knopp, C. L. Ober, L. G. Jovanovich, B. F. Polk, and the NICHD-Diabetes in Early Pregnancy Study Group. 1983. Malformations in infants of diabetic mothers: problems in study design. Prev. Med. 12:274-286.

45. Leslie, R. D. G., D. A. Pyke, P. N. John, and J. M. White. 1978 Hemoglobin $A_{l c}$ in diabetic pregnancy. Lancet. ii:958-959.

46. Miller, E., J. W. Hare, J. P. Cloherty, P. J. Dunn, R. E. Gleason, J. S. Soeldner, and J. L. Kitzmiller. 1981. Elevated maternal hemoglobin $A_{1 c}$ in early pregnancy and major congenital anomalies in infants of diabetic mothers. N. Engl. J. Med. 304:1331-1334.
47. Ylinen, K., P. Aula, U.-H. Stenman, T. Kesäniemi-Kuokkanen and K. Teramo. 1984. Risk of minor and major fetal malformations in diabetics with high hemoglobin $\mathrm{A}_{\mathrm{lc}}$ values in early pregnancy. Br. Med. J. 289:345-346.

48. Horii, K., G. Watanabe, and T. H. Ingalls. 1966. Experimental diabetes in pregnant mice: prevention of congenital malformations in offspring by insulin. Diabetes. 15:194-204.

49. Baker, L., J. M. Egler, S. M. Klein, and A. S. Goldman. 1981 Meticulous control of diabetes during organogenesis prevents congenital lumbosacral defect in rats. Diabetes. 30:955-959.

50. Eriksson, U. J., E. Dahlström, K. S. Larsson, and C. Hellerström. 1982. Increased incidence of congenital malformations in the offspring of diabetic rats and their prevention by maternal insulin therapy. Diabetes. 31:1-6. 